\title{
Wild sets and 2-ranks of class groups
}

\author{
by
}

P. E. Conner (Baton Rouge, La.), R. Perlis (Baton Rouge, La.) and K. SzYMiczEK (Katowice)

Dedicated to J. W. S. Cassels on the occasion of his 75th birthday

1. Introduction. The notion of two number fields $K$ and $L$ having equivalent Hilbert-symbol reciprocity laws was introduced in [PSCL] for the purpose of studying isomorphisms between the Witt rings $W(K)$ and $W(L)$. In that paper, the name reciprocity equivalence was used; however, in view of recent developments in which equivalences between higher-order reciprocity laws are discussed (see [CzSł]), these earlier equivalences are now more properly referred to as Hilbert-symbol equivalences. Here is the definition: A Hilbert-symbol equivalence between $K$ and $L$ is a pair of maps $(t, T)$ in which

$$
t: K^{*} / K^{* 2} \rightarrow L^{*} / L^{* 2}
$$

is an isomorphism of square-class groups, and

$$
T: \Omega_{K} \rightarrow \Omega_{L}
$$

is a bijection between the set of places of $K$ and those of $L$, preserving Hilbert-symbols in the sense that

$$
(a, b)_{P}=(t a, t b)_{T P}
$$

for all square-classes $a, b \in K^{*} / K^{* 2}$ and all places $P$ of $K$.

For the convenience of the reader, we recall that there is a Hilbert-symbol equivalence between number fields $K$ and $L$ if and only if $K$ and $L$ have the same level, the same number of real infinite places, and if there is a bijection between the dyadic places of $K$ and of $L$ so that the corresponding dyadic completions have the same degree over $\mathbb{Q}_{2}$ and also have the same local level. For details, see Theorem 1.5 of [Szym].

1991 Mathematics Subject Classification: Primary 11E81, 11R29.

The second author was partially supported by grant number LEQSF(1995-97)-RD-A09 from the Louisiana Educational Quality Support Fund. 
In the sequel, we frequently use the fact that, if $(t, T)$ is a Hilbert-symbol equivalence, then $T$ always maps real infinite places to real infinite places, finite places to finite places, and dyadic places to dyadic places. A proof is found in Lemma 4 of [PSCL].

Hilbert-symbol equivalences come in two types: tame and wild. The equivalence $(t, T)$ is said to be tame at the finite place $P$ if

$$
\operatorname{ord}_{P}(a) \equiv \operatorname{ord}_{T P}(t a)(\bmod 2)
$$

for all square classes $a \in K^{*} / K^{* 2}$; otherwise $(t, T)$ is wild at $P$. The set of finite wild places for $(t, T)$ is the wild set, denoted by Wild $(t, T)$. It can be empty, finite or infinite. When the wild set is empty, we say that $(t, T)$ is tame. Carpenter proved that when $K$ and $L$ are Hilbert-symbol equivalent via an equivalence having an infinite wild set then $K$ and $L$ are always Hilbert-symbol equivalent via another pair $(t, T)$ having a finite wild set (see [Carp]).

It is not difficult to see that the ideal class groups of $K$ and $L$ have the same 2-rank (we recall the definition below) when $K$ and $L$ admit a tame Hilbert-symbol equivalence. Hence, when the class groups of $K$ and $L$ have different 2-ranks, then any Hilbert-symbol equivalence $(t, T)$ between them is wild. This suggests that, as $(t, T)$ varies over all Hilbert-symbol equivalences between $K$ and $L$, there might be a relation between the size of the minimal wild set and the difference between the 2-ranks of the class groups. It turns out that such a relation exists, not only for class groups but also for narrow class groups, for $S$-class groups, and for narrow $S$-class groups. These relations will be established in this paper.

Once established, these relations can be restated to show that the difference of the 2-ranks of the tame kernels $K_{2}\left(\mathcal{O}_{K}\right)$ and $K_{2}\left(\mathcal{O}_{L}\right)$ is bounded above by the number of nondyadic places in the wild set of any Hilbertsymbol equivalence between $K$ and $L$.

The proofs given below are elementary. They replace earlier unpublished proofs that were heavily computational. We trust that the reader will not judge the value of the results by the simplicity of the proofs.

Here is our main result. Definitions and proofs are found in Section 2.

Proposition. Let $(t, T)$ be a Hilbert-symbol equivalence between number fields $K$ and $L$ with finite wild set $W=\operatorname{Wild}(t, T)$. Let $S \subset \Omega_{K}$ be any finite subset of places of $K$ containing all infinite places. Then

$$
\left|\mathrm{rk}_{2} C_{S}(K)-\mathrm{rk}_{2} C_{T S}(L)\right| \leq \#(W \backslash S)
$$

and

$$
\left|\mathrm{rk}_{2} C_{S}^{+}(K)-\mathrm{rk}_{2} C_{T S}^{+}(L)\right| \leq \#(W \backslash S) .
$$

From this Proposition, we obtain interesting corollaries by choosing $S$ to be the set of infinite places of $K$, or $S$ to be the set of dyadic and infinite 
places of $K$. These corollaries and the connections with $K$-groups are found in Section 3. Section 4 contains some illustrative examples.

We close this introduction by commenting that we are aware of some upper bounds for the minimal number of wild primes that fit nicely with the lower bounds proved here. Since the proofs of these upper bounds have a different character than the proofs here, these upper bounds will be published separately.

2. Proofs. We recall the definition of 2-rank. Let $G$ be a finite abelian group. The 2-rank of $G$, denoted $\mathrm{rk}_{2} G$, is the maximum of the numbers $j$ such that $G$ contains a subgroup of exponent 2 and order $2^{j}$. Restated, the 2-rank of $G$ is $r$ where $\left(G: G^{2}\right)=2^{r}$. Note that the 2-rank of $G$ coincides with the 2-rank of $G / G^{2}$.

For a number field $K, \Omega_{K}$ will denote the set of all places of $K$, finite and infinite, and $S \subset \Omega_{K}$ will denote a finite set of places always containing the infinite places. The $S$-class group $C_{S}(K)$ of $K$ is the quotient of the ordinary ideal class group, $C(K)$, by the subgroup of $C(K)$ generated by the ideal classes of all prime ideals $P$ that lie in the set $S$. If $S$ contains no finite primes, then $C_{S}(K)=C(K)$. We similarly define $C_{S}^{+}(K)$ to be the quotient of the narrow ideal class group $C^{+}(K)$ by the subgroup generated by narrow ideal classes of prime ideals in $S$.

Let $I(K)$ denote the ideal group of $K$. This is the free abelian group generated by the prime ideals of the ring of integers of $K$. Similarly, $I(L)$ denotes the ideal group of $L$. Since the set bijection $T: \Omega_{K} \rightarrow \Omega_{L}$ always maps finite places to finite places and infinite places to infinite places (see Lemma 4 in [PSCL]), it follows that $T$ canonically induces a group isomorphism between $I(K)$ and $I(L)$ that maps squares to squares, and hence induces a group isomorphism

$$
T_{\#}: I(K) / I(K)^{2} \rightarrow I(L) / I(L)^{2} .
$$

We begin with a lemma, which is simply a reformulation of the Proposition in the special case that $S$ contains the wild set $\operatorname{Wild}(t, T)$.

LEMMA. If $S$ is a finite set of places of $K$ containing the infinite places and containing the wild set $W=\operatorname{Wild}(t, T)$ of a Hilbert-symbol equivalence $(t, T)$ between $K$ and $L$, then $T$ induces isomorphisms

$$
C_{S}(K) / C_{S}(K)^{2} \cong C_{T S}(L) / C_{T S}(L)^{2}
$$

and

$$
C_{S}^{+}(K) / C_{S}^{+}(K)^{2} \stackrel{\cong}{\rightrightarrows} C_{T S}^{+}(L) / C_{T S}^{+}(L)^{2} .
$$


Proof. To begin, it follows from the definitions that, if $A$ and $B$ are integral ideals in $K$, then

$$
\operatorname{cl}_{S}(A)=\operatorname{cl}_{S}(B) \in C_{S}(K) / C_{S}(K)^{2}
$$

if and only if there is a global square class $x \in K^{*} / K^{* 2}$ with

$$
\operatorname{ord}_{P}(x)+\operatorname{ord}_{P}(A) \equiv \operatorname{ord}_{P}(B)(\bmod 2)
$$

for all $P$ outside $S$. Since $S$ contains the wild set $\operatorname{Wild}(t, T)$, the equivalence $(t, T)$ is tame at all primes $P$ outside $S$. Hence, for $P \notin S$,

$$
\operatorname{ord}_{T P}(t x) \equiv \operatorname{ord}_{P}(x)(\bmod 2) .
$$

Thus, $A$ and $B$ represent the same element in $C_{S}(K) / C_{S}(K)^{2}$ if and only if

$$
\operatorname{ord}_{T P}(t x)+\operatorname{ord}_{T P}\left(T_{\#}(A)\right) \equiv \operatorname{ord}_{T P}\left(T_{\#}(B)\right)(\bmod 2)
$$

for all $P \notin S$, which happens if and only if $T_{\#}(A)$ and $T_{\#}(B)$ represent the same element in $C_{T S}(L) / C_{T S}(L)^{2}$.

So $T_{\#}$ induces an isomorphism

$$
C_{S}(K) / C_{S}(K)^{2} \cong C_{T S}(L) / C_{T S}(L)^{2} .
$$

Moreover, since $T$ maps real infinite places of $K$ to real infinite places of $L$, and since $(t, T)$ preserves Hilbert symbols at these places, the square-class $x \in K^{*} / K^{* 2}$ is totally positive if and only if $t x \in L^{*} / L^{* 2}$ is totally positive. Hence $T_{\#}$ induces an isomorphism

$$
C_{S}^{+}(K) / C_{S}^{+}(K)^{2} \stackrel{\cong}{\rightrightarrows} C_{T S}^{+}(L) / C_{T S}^{+}(L)^{2},
$$

proving the Lemma.

We now prove the Proposition, stated in the Introduction.

Proof of Proposition. If $W \backslash S$ is empty, then $W \subset S$ and the Proposition follows immediately from the Lemma. So now assume that $W \backslash S$ is nonempty.

Let $\mathcal{W}$ denote the subgroup of $C_{S}(K) / C_{S}(K)^{2}$ generated by $\mathrm{cl}_{S}(P)$ for all $P \in W \backslash S$. Similarly, $\mathcal{W}^{\prime}$ is the subgroup of $C_{T S}(L) / C_{T S}(L)^{2}$ generated by all the $\operatorname{cl}_{T S}(T P)$ for $P \in W \backslash S$. Then

$$
1 \rightarrow \mathcal{W} \rightarrow C_{S}(K) / C_{S}(K)^{2} \rightarrow C_{S \cup W}(K) / C_{S \cup W}(K)^{2} \rightarrow 1
$$

and

$$
1 \rightarrow \mathcal{W}^{\prime} \rightarrow C_{T S}(L) / C_{T S}(L)^{2} \rightarrow C_{T S \cup T W}(L) / C_{T S \cup T W}(L)^{2} \rightarrow 1
$$

are both short exact sequences of elementary abelian 2 -groups. Therefore the 2-rank of the middle term is the sum of the 2-ranks of its two neighboring terms, giving

$$
\operatorname{rk}_{2} C_{S}(K)=\mathrm{rk}_{2}(\mathcal{W})+\operatorname{rk}_{2} C_{S \cup W}(K)
$$


and

$$
\mathrm{rk}_{2} C_{T S}(L)=\mathrm{rk}_{2}\left(\mathcal{W}^{\prime}\right)+\mathrm{rk}_{2} C_{T S \cup T W}(L) .
$$

However, since $S \cup W$ contains $W$, the Lemma shows that $C_{S \cup W}(K)$ and $C_{T S \cup T W}(L)$ have the same 2-rank. Thus we arrive at

$$
\mathrm{rk}_{2} C_{S}(K)-\mathrm{rk}_{2} C_{T S}(L)=\mathrm{rk}_{2}(\mathcal{W})-\mathrm{rk}_{2}\left(\mathcal{W}^{\prime}\right) .
$$

We now observe that $0 \leq \operatorname{rk}_{2}(\mathcal{W}) \leq \#(W \backslash S)$ and $0 \leq \operatorname{rk}_{2}\left(\mathcal{W}^{\prime}\right) \leq \#(W \backslash S)$. With (1), it follows that

$$
\left|\mathrm{rk}_{2} C_{S}(K)-\mathrm{rk}_{2} C_{T S}(L)\right|=\left|\operatorname{rk}_{2}(\mathcal{W})-\operatorname{rk}_{2}\left(\mathcal{W}^{\prime}\right)\right| \leq \#(W \backslash S) .
$$

The same argument applies mutatis mutandis to the narrow $S$-class groups, proving the Proposition.

3. Corollaries and connections with $K$-theory. For the record, we note

Corollary 1. If $K$ and $L$ are tamely Hilbert-symbol equivalent, then

$$
C(K) / C(K)^{2} \cong C(L) / C(L)^{2} .
$$

Proof. This is the case of an empty wild set $W=\emptyset$. Apply the Proposition.

Apart from the inequality $\operatorname{rk}_{2} C_{S}^{+}(K) \geq \operatorname{rk}_{2} C_{S}(K)$, we do not know any general relations between $\operatorname{rk}_{2} C_{S}(K)$ and $\operatorname{rk}_{2} C_{S}^{+}(K)$. It is worthwhile pointing out

Corollary 2. Let $(t, T)$ be a Hilbert-symbol equivalence between $K$ and $L$, and let $d(K, L)$ denote the larger of $\left|\mathrm{rk}_{2} C(K)-\mathrm{rk}_{2} C(L)\right|$ and $\left|\mathrm{rk}_{2} C^{+}(K)-\mathrm{rk}_{2} C^{+}(L)\right|$. Then

$$
d(K, L) \leq|\operatorname{Wild}(t, T)| .
$$

Pr o of. Apply the Proposition to the set $S$ of infinite places of $K$.

The number $d(K, L)$ is thus a lower bound on the size of the wild set for any Hilbert-symbol equivalence between $K$ and $L$.

This raises the question: Does $d(K, L)$ equal the minimal number of wild primes that can occur in a Hilbert-symbol equivalence between $K$ and $L$ ?

Let $D$ denote the set of dyadic and infinite places of $K$ and let $D^{\prime}$ denote the set of dyadic and infinite places of $L$. Given a Hilbert-symbol equivalence $(t, T)$ from $K$ to $L$, then $D^{\prime}=T(D)$, by [PSCL]. Let Wild odd $(t, T)$ denote the set of nondyadic places in Wild $(t, T)$. Let $d_{D}(K, L)$ denote the larger of $\left|\mathrm{rk}_{2} C_{D}(K)-\mathrm{rk}_{2} C_{D^{\prime}}(L)\right|$ and $\left|\mathrm{rk}_{2} C_{D}^{+}(K)-\mathrm{rk}_{2} C_{D^{\prime}}^{+}(L)\right|$. We have

Corollary 3. For any Hilbert-symbol equivalence $(t, T)$ from $K$ to $L$ with finite wild set

$$
d_{D}(K, L) \leq\left|\operatorname{Wild}_{\text {odd }}(t, T)\right|
$$


Proof. Set $S=D$ in the Proposition.

We ask whether $d_{D}$ equals the least number of nondyadic wild primes that can appear in any Hilbert-symbol equivalence between $K$ and $L$.

The wild set is not at all arbitrary. In fact, the following corollary shows that the ideal classes of the wild primes generate the ideal class group $C(K)$ when $C(K)$ is a 2-group and when $K$ is Hilbert-symbol equivalent to a field $L$ having odd class number.

COROLlary 4. Let $(t, T)$ be a Hilbert-symbol equivalence between $K$ and $L$ with finite wild set $W$. If the class number $h_{L}$ is odd, then the subgroup of the ideal class group $C(K)$ generated by ideal classes of the wild primes contains the 2-Sylow subgroup of $C(K)$.

P r o of. Suppose $h_{L}$ is odd. Let $S$ be the union of the wild set with the set of all infinite places of $K$. Since $S$ contains $W$, it follows from the Proposition that $\operatorname{rk}_{2} C_{S}(K)=\operatorname{rk}_{2} C_{T S}(L)$, which is 0 since $C_{T S}(L)$ is a quotient of the group $C(L)$ having odd order. Since the odd-order group $C_{S}(K)$ is the quotient of $C(K)$ by the subgroup generated by the wild primes, it follows that the wild primes generate at least the 2-Sylow subgroup of $C(K)$.

We remark that Corollary 4 and its proof remain true if we replace the ideal classes, class groups and class numbers by the corresponding narrow ideal classes, narrow class groups and narrow class numbers.

Next, we connect the Proposition with the tame kernel, $K_{2}\left(\mathcal{O}_{K}\right)$. In [CoHu], Lemma 25.1, it was pointed out that combining (6.1) and (6.3) of Tate's paper [Tate] produces the following formula for the 2-rank of the tame kernel:

$$
\mathrm{rk}_{2} K_{2}\left(\mathcal{O}_{K}\right)=r_{1}(K)-1+g_{2}(K)+\mathrm{rk}_{2} C_{D}(K) .
$$

We refer to (3) as Tate's 2-rank formula. As usual, $r_{1}(K)$ denotes the number of real infinite places of $K$, and $g_{2}(K)$ denotes the number of dyadic places of $K$. A Hilbert-symbol equivalence $(t, T)$ from $K$ to $L$ always preserves $r_{1}$ and $g_{2}$ (see [PSCL]). Thus, when $K$ and $L$ are Hilbert-symbol equivalent, then

$$
\left|\mathrm{rk}_{2} K_{2}\left(\mathcal{O}_{K}\right)-\operatorname{rk}_{2} K_{2}\left(\mathcal{O}_{L}\right)\right|=\left|\mathrm{rk}_{2} C_{D}(K)-\mathrm{rk}_{2} C_{D^{\prime}}(L)\right| .
$$

COROLlary 5. When $(t, T)$ is a Hilbert-symbol equivalence from $K$ to $L$ with finite wild set, then

$$
\left|\mathrm{rk}_{2} K_{2}\left(\mathcal{O}_{K}\right)-\mathrm{rk}_{2} K_{2}\left(\mathcal{O}_{L}\right)\right| \leq\left|\operatorname{Wild}_{\text {odd }}(t, T)\right| \text {. }
$$

Proof. This is just (4) combined with Corollary 3.

We end this section by observing that one-half of the nondyadic primes are often excluded from being wild. Suppose that $K$ does not have level 1. Then -1 is a local nonsquare for an infinite set of prime ideals of $\mathcal{O}_{K}$ of 
Dirichlet density one-half. Thus, at any nondyadic prime $P$ at which -1 is a local nonsquare, the four local square classes at $P$ are represented by $\{1,-1, \pi,-\pi\}$, where $\pi$ is a local uniformizer at $P$. Let $(t, T)$ be any Hilbertsymbol equivalence from $K$ to some other field $L$. By [PSCL], Lemma 4, the global square-class isomorphism $t$ in $(t, T)$ induces a compatible local square-class isomorphism $t_{P}$ from $K_{P}^{*} / K_{P}^{* 2}$ to $L_{T P}^{*} / L_{T P}^{* 2}$, and both $t$ and $t_{P}$ necessarily map the square-class of -1 to the square-class of -1 . If $a$ is a global square-class in $K^{*} / K^{* 2}$, then $\operatorname{ord}_{P}(a) \equiv 0(\bmod 2)$ if and only if $a$ is represented by 1 or -1 locally at $P$, if and only if $t a=t_{P} a$ is represented by 1 or -1 locally at $T P$. It follows that $\operatorname{ord}_{P}(a) \equiv \operatorname{ord}_{T P}(t a)(\bmod 2)$ for all global square-classes $a$. This forces $(t, T)$ to be tame at $P$. So one-half of the finite nondyadic primes are not in the wild set of any Hilbert-symbol equivalence starting from (or ending at) $K$.

4. Examples. Every quadratic field is Hilbert-symbol equivalent to exactly one of the following seven fields:

$$
\mathbb{Q}(\sqrt{-1}), \quad \mathbb{Q}(\sqrt{ \pm 2}), \quad \mathbb{Q}(\sqrt{ \pm 7}), \quad \mathbb{Q}(\sqrt{ \pm 17}),
$$

(see [Carp] and [Czog]). Since Hilbert-symbol equivalence preserves level and degree over $\mathbb{Q}$, the Gaussian field $\mathbb{Q}(\sqrt{-1})$ is Hilbert-symbol equivalent only to itself. Given a square-free integer $d \neq \pm 1$, the quadratic field $K=\mathbb{Q}(\sqrt{d})$ is Hilbert-symbol equivalent to its canonical cousin, which is defined to be $\mathbb{Q}(\sqrt{\operatorname{sign}(d) \cdot m})$ where $m$ is computed as follows: when $|d| \equiv 1(\bmod 8)$ then $m=17$, when $|d| \equiv 2,3,5,6(\bmod 8)$ then $m=2$, and when $|d| \equiv 7$ $(\bmod 8)$ then $m=7$.

Six of the canonical fields listed in (5) have class number 1 and therefore have class groups with 2-rank 0 , the exception being $\mathbb{Q}(\sqrt{-17})$, which has a cyclic class group of order 4 and 2-rank 1. Any field that is Hilbert-symbol equivalent to the exceptional field $\mathbb{Q}(\sqrt{-17})$ has class number divisible by 4 (the class number is even by [Szym], and the class number is not congruent to 2 modulo 4 by [CoHu], (19.6)).

Gauss proved that the 2-rank of the narrow class group of a quadratic field $K$ is $n-1$ where $n$ is the number of rational prime numbers dividing the discriminant of $K \mid \mathbb{Q}$. The narrow class group $C^{+}(K)$ has the same 2-rank as the ordinary ideal class group $C(K)$ except when $K$ is real and $-1 \in \mathbb{Q}$ is not the norm of any element in $K$, in which case $\mathrm{rk}_{2} C(K)=n-2$ (see [CoHu], (18.3)).

Thus, excluding $\mathbb{Q}(\sqrt{-1})$, six of the canonical fields listed in (5) are Hilbert-symbol equivalent to a quadratic field $K$ whose class group has 2rank as large as we wish. Let $(t, T)$ be a Hilbert-symbol equivalence between $K$ and one of the six fields in $(5)$, excluding $\mathbb{Q}(\sqrt{-1})$. By our Proposition, the number of wild primes of $(t, T)$ is bounded below by the difference between 
$\mathrm{rk}_{2} C(K)$ and the 2-rank of the class group of $K$ 's canonical cousin. Thus, the number of wild primes of $(t, T)$ will normally be no smaller than

$$
\mathrm{rk}_{2} C(K)-0=n-1,
$$

the only exception being when $K$ is Hilbert-symbol equivalent to $\mathbb{Q}(\sqrt{-17})$, in which case the number of wild primes will be no less than $\operatorname{rk}_{2} C(K)-$ $1=n-2$. As above, $n$ denotes the number of prime numbers dividing the discriminant of $K \mid \mathbb{Q}$.

For our next example, observe that $K=\mathbb{Q}(\sqrt{2})$ and $L=\mathbb{Q}(\sqrt{3})$ are Hilbert-symbol equivalent and each has class number 1 . However, the narrow class number of $K$ is 1 while the narrow class number of $L$ is 2 . Thus, the 2-ranks of the narrow class groups differ by 1 , so every Hilbert symbol equivalence between $K$ and $L$ must have at least one wild prime.

Next, consider $K=\mathbb{Q}(\sqrt{-10})$. The class group of $K$ has order 2 and 2 -rank 1 . There is a unique dyadic prime, $\wp$, in $\mathcal{O}_{K}$ and

$$
2 \mathcal{O}_{K}=\wp^{2} .
$$

Since 2 is not a norm from $K \mid \mathbb{Q}$, it follows that $\wp$ is not itself principal. So $\operatorname{cl}(\wp)$ generates $C(K)$. Let $D$ consist of the dyadic prime $\wp$ together with the complex infinite place of $K$. Then $C_{D}(K)$ is trivial. If $(t, T)$ is a tame Hilbert-symbol equivalence from $K$ to some other quadratic field $L$, then $T_{\wp}$ is the unique dyadic prime of $L$ and $\operatorname{rk}_{2} C_{T D}(L)=\mathrm{rk}_{2} C_{D}(K)=0$. Hence, $\operatorname{cl}(T \wp) \in C(L)$ generates a cyclic subgroup of $C(L)$ which must contain the 2-Sylow subgroup of $C(L)$.

For our final example, let $E=\mathbb{Q}(\sqrt{-14})$. The class group $C(E)$ is known to be cyclic of order 4 . Let $F=\mathbb{Q}(\sqrt{-2})$. Then $E$ and $F$ are Hilbert-symbol equivalent, and $h_{F}=1$. Let $(t, T)$ be a Hilbert-symbol equivalence from $E$ to $F$. We let the reader use the methods of this paper to verify the following statements:

a. The ideal classes of the wild primes generate $C(E)$.

b. There must always be at least one nondyadic wild prime.

c. There is always at least one nondyadic wild prime $P$ whose ideal class generates $C(E)$.

d. Let $P$ be a nondyadic wild prime generating $C(E)$, from statement c above, and let $p$ be the rational prime number lying below $P$. Then $p \equiv 5$ $(\bmod 8)$ and $(7, p)_{p}=-1$.

This example illustrates yet again that wild primes are far from arbitrary!

We conclude these examples by recalling that Czogała has classified quadratic fields up to tame equivalence. See [Czog]. 


\section{References}

[Carp] J. P. Carpenter, Finiteness theorems for forms over global fields, Math. Z. 209 (1992), 153-166.

[CoHu P. E. Conner and J. Hurrelbrink, Class Number Parity, Ser. Pure Math. 8, World Scientific, Singapore, 1988.

[Czog] A. Czogała, On reciprocity equivalence of quadratic number fields, Acta Arith. 58 (1991), 27-46.

[CzSł] A. Czogała and A. Sładek, Higher degree Hilbert symbol equivalence of number fields, Tatra Mt. Math. Publ. 11, to appear.

[PSCL] R. Perlis, K. Szymiczek, P. E. Conner and R. Litherland, Matching Witts with global fields, in: Contemp. Math. 155, Amer. Math. Soc., 1994, 365-387.

[Szym] K. Szymiczek, Witt equivalence of global fields, Comm. Algebra 19 (1991), $1125-1149$.

[Tate] J. Tate, Relations between $K_{2}$ and Galois cohomology, Invent. Math. 36 (1976), $257-274$

Department of Mathematics

Louisiana State University

Baton Rouge, Louisiana 70803-4918

U.S.A.

E-mail: perlis@math.lsu.edu
Institute of Mathematics Silesian University Bankowa 14

40-007 Katowice, Poland

E-mail: szymiczek@gate.math.us.edu.pl 